\title{
Performance Optimization of Ad Hoc on Demand Vector Routing Protocol in Cognitive Radio Network
}

\author{
Shruti Singh Roy ${ }^{1}$, P. L. Zade ${ }^{2}$ \\ ${ }^{1}$ Electronics \& Telecommunication Y.C.C.E Nagpur, India \\ ${ }^{2}$ Electronics \& Telecommunication Y.C.C.E Nagpur, India
}

Received: 12 February 2018; Accepted: 08 March 2018; Published: 08 May 2018

\begin{abstract}
Mobile Ad Hoc Network has given importance in last few years by researchers. Implementations of Ad Hoc network in cognitive radio environment are emerging kind of wireless network $[1,5]$. The communication is done by the secondary user in the free frequency band available in the spectrum of the primary user. In this paper, we have projected proficient routing solution by the maintenance of the link for AOMDV protocol for cognitive radio ad hoc Network. We have used ns2 Simulator for simulations. The link maintenance makes the system more efficient that is evaluated by simulation and comparing it with a classical routing protocol.

Index Terms: Proactive routing protocol, reactive routing protocol, DSDV, AODV, AOMDV, LMAOMDV.

(C) 2018 Published by MECS Publisher. Selection and/or peer review under responsibility of the Research Association of Modern Education and Computer Science
\end{abstract}

\section{Introduction}

The concept of wireless network was started in the eighteenth century and the technology is growing every day. The wireless networks are classified as

a) Infrastructure network

b) Ad Hoc Network

c) Hybrid network

The combination of Ad Hoc Network and infrastructure network is known as hybrid network.

\footnotetext{
* Corresponding author.

E-mail address: shruti_singhroy@rediffmail.com
} 


\section{a) Infrastructure network}

These types of networks are generally established by Wi-Fi router device communication using single point that is centrally located and connected to each device [2].

\section{b) Ad Hoc Network}

In Ad Hoc networks, centralizes access point is not required. The devices are directly getting connected to each other. All the nodes in the ad-hoc network are random in environment. Each node acts as a router and is able to find its new part and maintained routes for transmitting packets. This transmission of data packets are done with help of routing protocol. Some of the standard routing protocols are discussed below.

\section{Routing protocol}

The process of finding path or route between originator and target is known as routing. The routing protocol is used whenever packets are needed to be transmitted from source to destination via different nodes [8]. Routing protocols are proposed in various forms and in various parameters that affect the communications such as unicast routing, speed power consumption, residual energy, quality of service, network over and make more. Depending upon these challenges the routing protocols are divided as follows

i. reactive or on-demand routing protocol

ii. proactive or table-driven routing protocol and

iii. hybrid

Under these types, there are various routing protocols to overcome these challenges and to design efficient network.

\section{Ad Hoc on demand distance vector routing protocol}

AODV is reactive type protocol. Ad Hoc on-demand protocol is capable of setting up forward path and reversed path. Then it uses route discovery mechanism for transmission of packets to receiver whenever needed. AODV adopts a traditional method of table entry between intermediate notes with a minimum number of the broadcast that is it takes the shortest path for communication between sources to a destination [6]. AODV uses the source IP address and IP header for unique identification. Source node initiates part Discovery process by broadcasting on route request (RREQ) packets to neighbouring nodes [9]. The neighbouring nodes will again send the route request to the next and will continue to broadcast the message until the destination reached. To make it a loop-free and overwriting of information, every node maintained two separate counter sequence number and broadcast id as shown in Fig 1.

\begin{tabular}{|c|}
\hline \hline destination \\
\hline sequence number \\
\hline hopcount \\
\hline expiration_timeout \\
\hline nexthop \\
\hline \hline
\end{tabular}

Fig.1. Structure of routing table entry of AODV 
The address and broadcast id are unique defines the route request message. For every route request message broadcast id is incremented to initiate the source node. Sometimes there will be flooding of route request between intermediate nodes. During flooding, the nodes will only keep the first receive message and will discard the other. The first copy of Broadcast package is having the address of the neighbours that is recorded by the intermediate nodes before forwarding. Due to this process reversed parties for were formed back to source automatically. When there is a link failure, a message is sent reverse to the source node to inform about the failure. Then the source node will re-initiate the route discovery process. AODV choose least congestion route rather than shortest path, unicast and multicast packet transmission.

\section{AOMDV ( Ad Hoc on Demand Multipath Distance Vector Routing Protocol)}

AODV is further modified and many challenges are done as AOMDV for computing multipath loop-free and route smash between source destination pair. With the help of multipath, the protocols can switch to another part when there is linked disjoint path. It with reduce route discovery retransmission. If a single path fails, there will be no need for route discovery process, as it stores multipath to a destination using single route discovery [10]. When the entire path to the destination fails then only is route discovery process starts. Whereas for single part protocol every time new route discovery process is required for the source to destination.

\begin{tabular}{|c|}
\hline \hline destination \\
\hline sequence number \\
\hline advertised_hopcount \\
\hline expiration_timeout \\
\hline route_list \\
$\left\{\left(\right.\right.$ nexthop $_{1}$, hopcount $\left._{1}\right)$, \\
$\left(\right.$ nexthop $_{2}$, hopcount $\left.\left._{2}\right), ..\right\}$ \\
\hline \hline
\end{tabular}

Fig.2. Structure of routing table entry of AOMDV

In the previous protocol, a reversed path has been created to source from destination through the same path of route request in the first hope which is unique. While keeping all such copies of reverse path, this will cause a loop in routing protocol. Fig 2 shows who the routing table is maintained when the updated data packets are received.

The multipath routing process will find the disjoint routes. The node does not refuse the repeat route request. Every repeated route request arriving through different intermediate node and neighbor of the source will tell about the link disjoint route. Any two repeated route request will not be able to broadcast via a same intermediate node to neighbors to a source. The destination will only send a reply to route request when it will come through a unique neighbor. The route request will travel through intermediate nodes and takes the different overturn path to the source to find the linked dislodge route [7]. The AOMDV will allow the inbetween nodes to reply the route request, is the main feature.

\section{DSDV (Distance Sequence Distance Vector)}

Distance vector algorithm maintains particular distance for each destination. The source node treats the neighbor as the next hope for every packet. The repetition of next hope is so chosen so that the path from source to destination became the shortest path. To keep the record of each hop count come every node maintains the list in form of the table. So DSDV is the table driven protocol with the information of every node in the network [11].

The DSDV is based on Distributed Bellman-Ford Algorithm (DBF). The data is transmitted between source and destination with reference to the routing table [11]. To maintain the time between each data transmits the 
tables are updated periodically. The data is routed through either layer 2 MAC or layer 3 Address [3]. It follows some particular pattern before broadcast. The data transferred will have one sequence number followed by receiver address the shortest path to the receiver. The routing table contains the information regarding transmission and reception. When the data is transmitted the receiver adding the positive feedback path and by each incrimination, the path will be created to each destination.

\section{LMAOMDV (Link Maintenance Ad Hoc on Demand Multi Path Distance Vector)}

We have designed a new routing protocol i.e. LMAOMDV which is reactive routing protocol that is better than the proactive protocol. The below flow diagram i.e. Fig 3 shows the working of the link maintenance ad hoc on demand multipath distance vector routing protocol. This is a very extremely, dexterous and scalable distributed routing algorithm which is based on link maintenance and link reversal. LMAOMDV has two essential functions

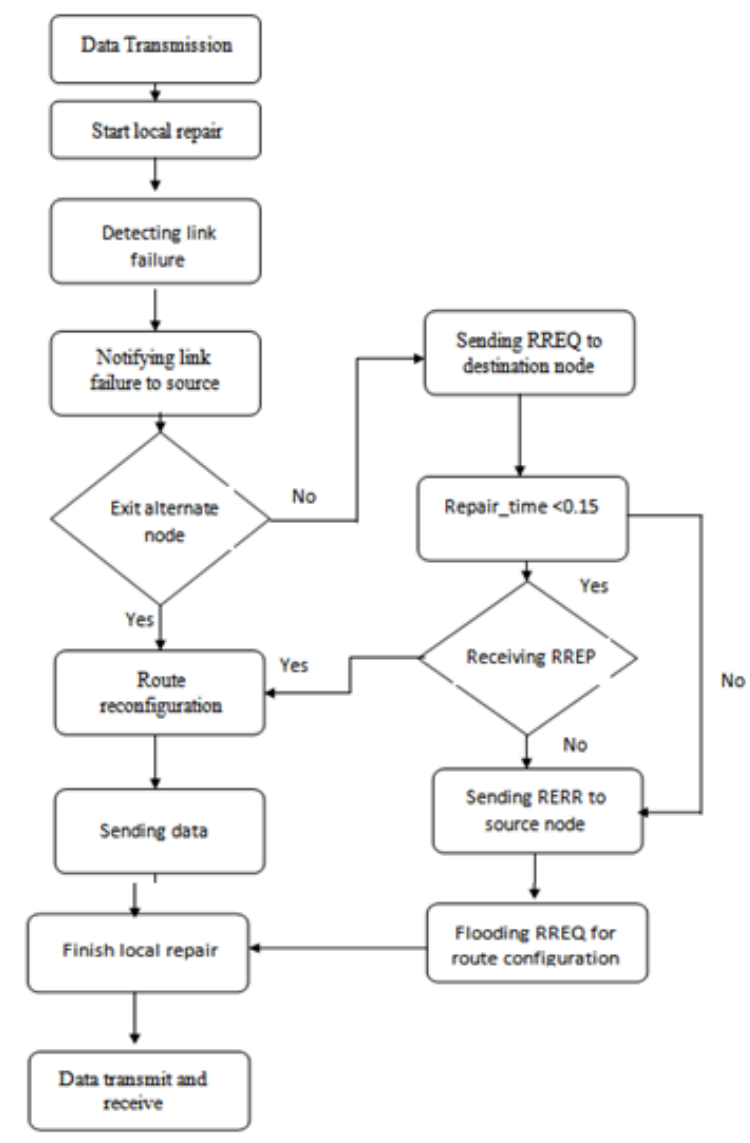

Fig.3. flow diagram of LMAOMDV 

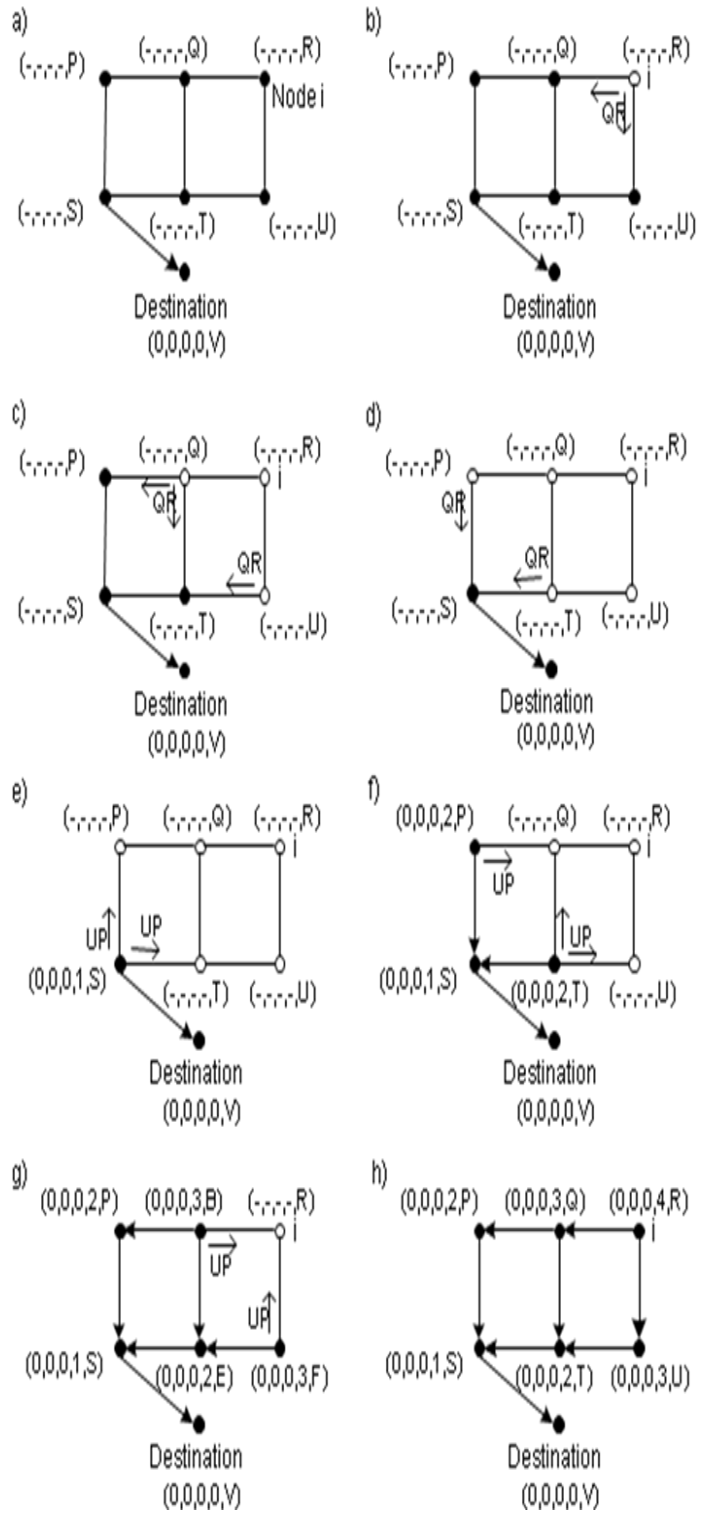

Fig.4. Communications between nodes

\section{1) Route discovery \\ 2) Route maintenance}

For finding the route, query packets are sent to every node. Accordingly, the heights of the destinations and the entire node are decided. At the start of the communication, the destination is set to zero and all the other nodes as NULL. As we know that the water flows from higher level to lower level. In a similar way, the data will flow from higher height to lower height. The below flowchart will give the detailed idea of LMAOMDV. 
When the source node will start the data transmission, before that they will create a route discovery process. It will create a query packet that will propagate between neighbours. In the meantime, the route request flag will get set. Whenever any nodes get the query packet it will set the route request flag and broadcast the packet further, until it gets the neighbour of the destination.

The neighbour of the target knows about it will generate an update packet and also sets its height. During this process, the route request flag will become zero. The update packet will decide the path and the source will know about the different multipath present.

\section{Simulation parameters}

Following are the simulation which we used for the design of cognitive radio network.

Table 1. Simulation parameters

\begin{tabular}{|c|c|}
\hline Parameter & Quantity \\
\hline Propagation & Two Ray Ground \\
\hline Network & Cognitive Radio Network \\
\hline Queue & DropTail \\
\hline Antenna & Omni Directional Antenna \\
\hline Max packet in queue & 50 \\
\hline Number of nodes & 50 \\
\hline Routing Protocols & AODV, AOMDV, DSDV, \\
LMAOMDV
\end{tabular}

In this research, we use propagation model as two ray ground propagation model. A queue is drop tail because when a number of packets will be in the wait in line for certain duration of time if queue limit ends the last packet will be dropped. A number of packets in the queue are 50 and the total number of nodes in this simulation is 50 . The routing protocols play a significant role in the wireless system. So to provide a suitable route to the nodes here we have used reactive and proactive routing protocols such as AODV, AOMDV, DSDV, and LMAOMDV. The total area of the cognitive area network is $600 \mathrm{~m} \mathrm{X} 600 \mathrm{~m}$.

\section{Results}

Cognitive radio network senses the empty channel and then it provides to the needy user without any interference of other users. After calculating performance parameters of the cognitive area network, here we can check the status of the system. Fig 5 represents the average throughput, PDR, and EED of AODV routing protocol. Fig. 6 represents the average throughput, PDR, and EED of AOMDV routing protocol 

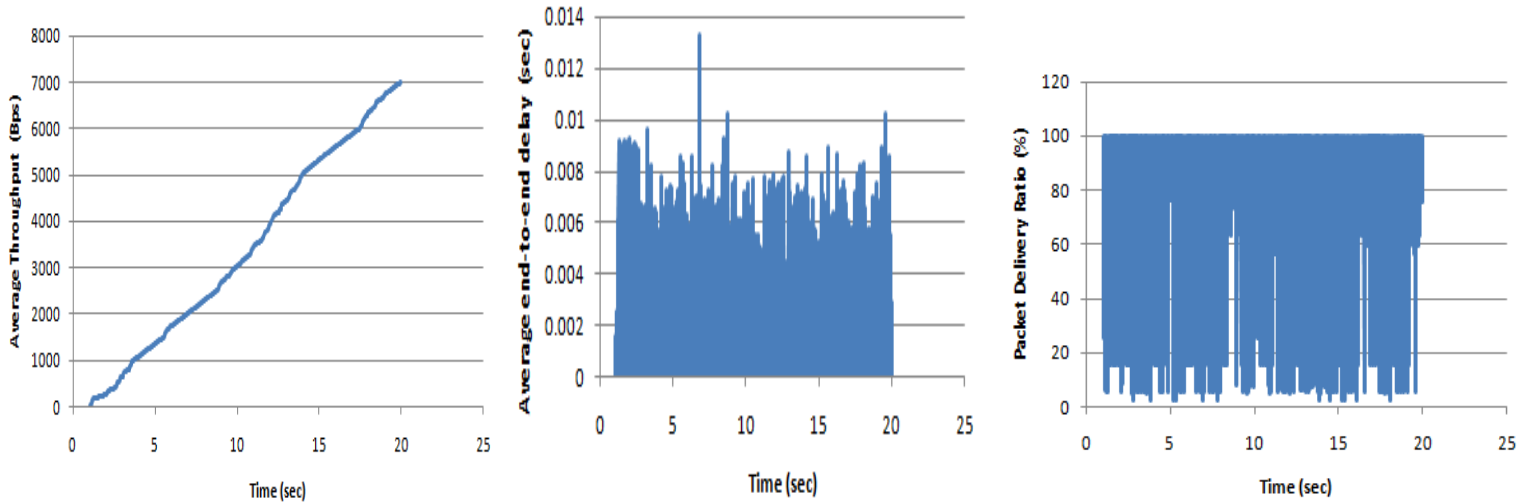

Fig.5. Average throughput, PDR, and EED of AODV routing protocol
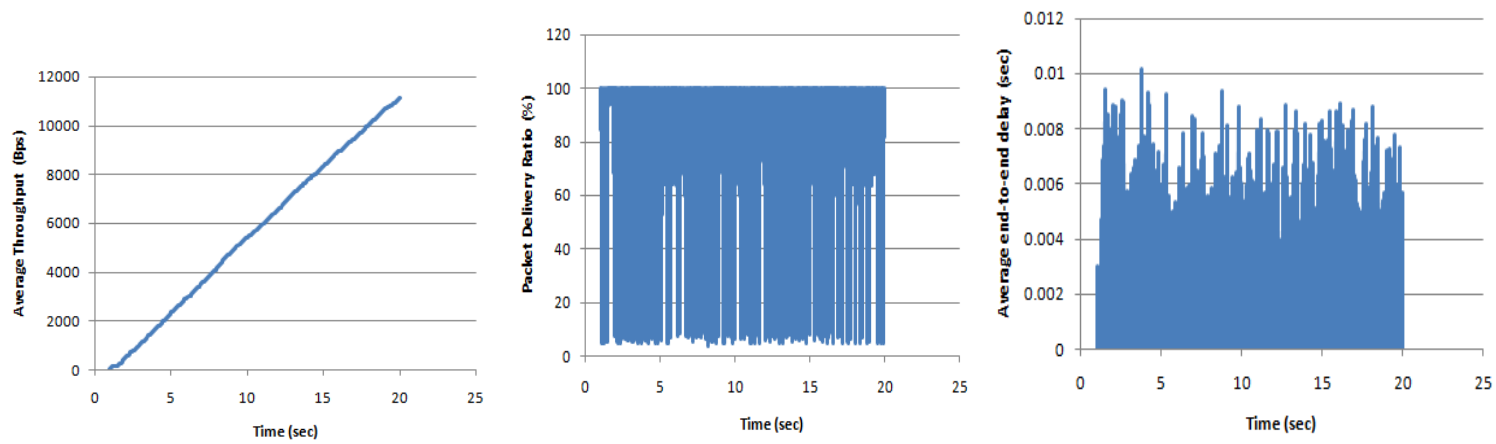

Fig.6. Average throughput, PDR, and EED of AOMDV routing protocol
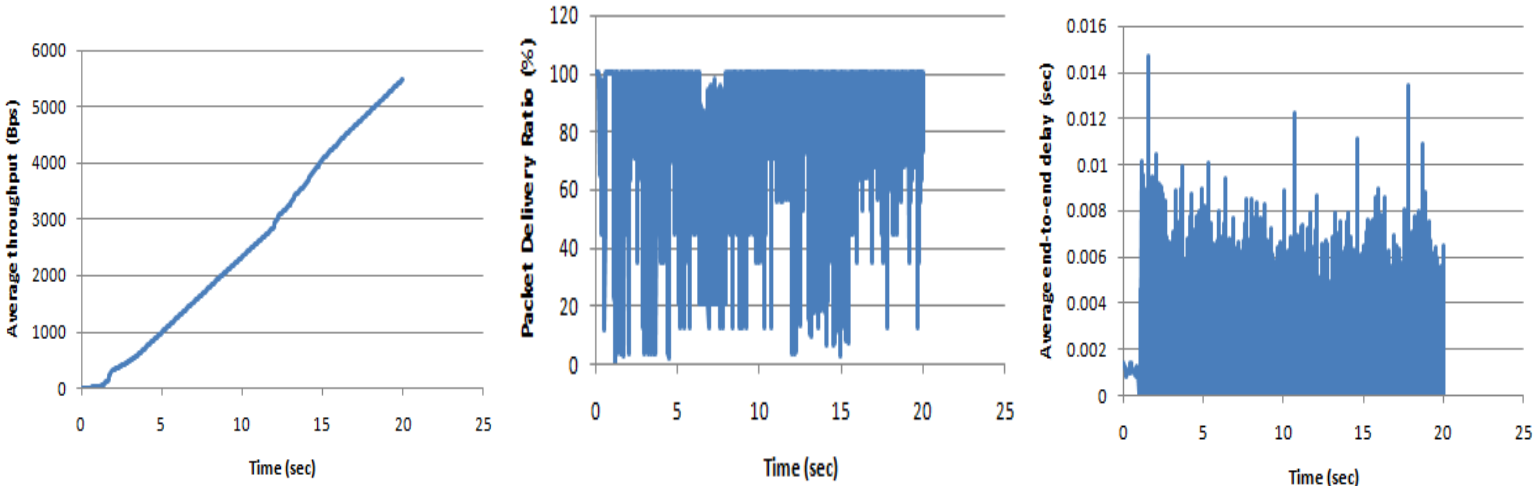

Fig.7. Average throughput, PDR, and EED of DSDV routing protocol 

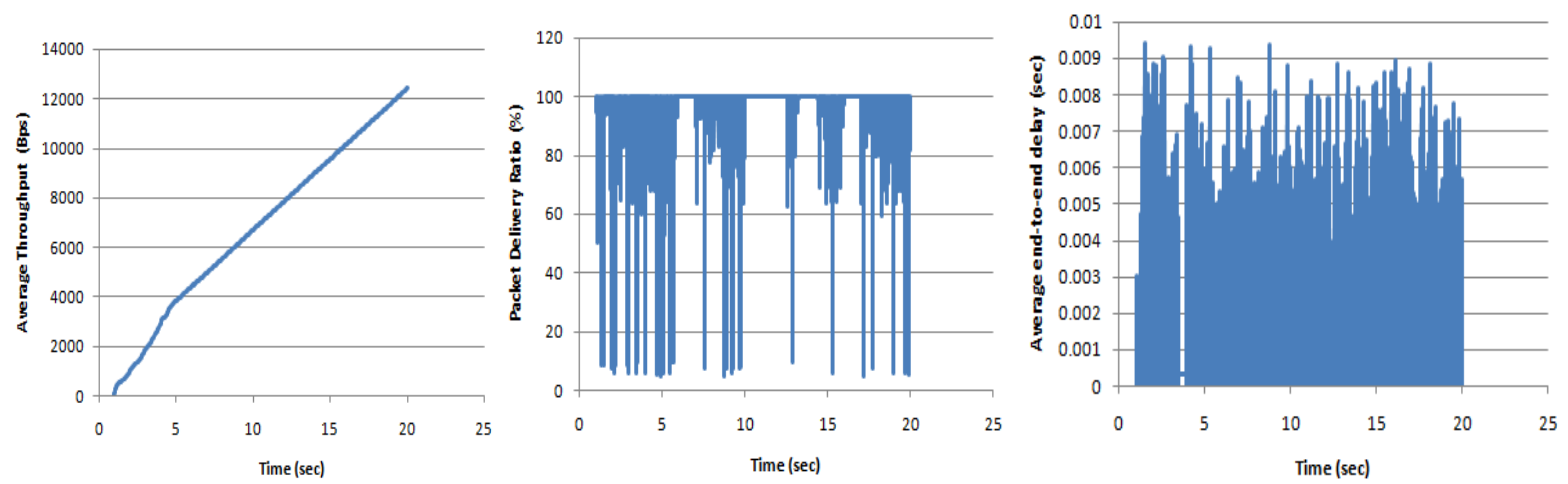

Fig.8. Average throughput, PDR, and EED of LMAOMDV routing protocol

Table 2. Comparison of performance parameters

\begin{tabular}{|c|c|c|c|c|}
\hline $\begin{array}{c}\text { Performance } \\
\text { Parameter }\end{array}$ & AODV & AOMDV & DSDV & LMAOMDV \\
\hline $\begin{array}{c}\text { Average } \\
\text { throughput (Bps) }\end{array}$ & 3525.80 & 5767.60 & 2787.23 & 6983.11 \\
\hline PDR (\%) & 92.72 & 94.81 & 87.86 & 98.11 \\
\hline $\begin{array}{c}\text { Average end to } \\
\text { end delay (mSec) }\end{array}$ & 1.031 & 1.023 & 1.096 & 1.004 \\
\hline
\end{tabular}

Table 2 shows the comparison of the functioning parameter of the CRN system for different routing protocols. Here we can see that the average throughput of the improved AOMDV routing protocol is better than that of other existing standard routing protocols. The similar case happens for packet delivery ratio. With enhanced average throughput the packet delivery ratio is also improved. But when you check for the average end to end delay the LMAOMDV gives the lowest delay than other three routing protocols. After LMAOMDV, AOMDV routing protocol is giving better results as compared to AODV and DSDV routing protocols. Here DSDV routing protocol provides worst results because it is proactive routing protocol which continuously upgrades their routing table without any reason and so it takes more time for its functioning.

\section{Conclusions}

The designed improved routing protocol provides better performance on cognitive radio network as compared to AODV, AOMDV, and DSDV routing protocols in terms of average throughput, average end to end delay and packet delivery ratio. LMAOMDV routing protocol is reactive routing protocol and is better than proactive routing protocol because it makes routing table when the user demands.

Here we conclude that improved AOMDV routing protocol works better for this designed cognitive radio network in terms of calculated performance parameters. 


\section{References}

[1] Wang and K. R. Liu, "Advances in cognitive radio networks: A survey,” IEEE J. Sel. Topics Signal Process., vol. 5, no. 1, pp. 5-23, Feb. 2011.

[2] Rashmi Mule and Bharati Patil, " Proactive Source Routing Protocol for Opportunistic Data Forwarding in MANETs", International Conference on Automatic Control and Dynamic Optimization Techniques (ICACDOT) International Institute of Information Technology (I2IT), Pune, 2016.

[3] Yean-Fu Wen and Wanjiun Liao, "Spectrum Section Preallocation for Cooperative Sensing and Transmission in Cognitive Radio Ad Hoc Networks", IEEE TRANSACTIONS ON VEHICULAR TECHNOLOGY, VOL. 66, NO. 10, OCTOBER 2017.

[4] Md E Ahmed, D. I. Kim , J. Y. Kim, and Yoan Shin," Energy-Arrival-Aware Detection Threshold in Wireless-Powered Cognitive Radio Networks", IEEE TRANSACTIONS ON VEHICULAR TECHNOLOGY, VOL. 66, NO. 10, OCTOBER 2017.

[5] Lee, W. Y, Akyildiz, I. F., and Chowdhury, K. R., "CRAHNs: Cognitive Radio Ad Hoc Networks," Ad Hoc Networks (Elsevier) Journal, to appear, vol. 7, July 2009

[6] Prof. S. Mahamuni, V. Mishra and V. M. Wadhai ,"Performance Evaluation of AODV Routing Protocol in Cognitive Radio Ad-hoc Network", International Journal of Wireless \& Mobile Networks (IJWMN) Vol. 3, No. 5, October 2011.

[7] S. Selvakanmani and Dr. M. Sumathi, "Overview and literature survey on routing protocols for mobile cognitive radio ad hoc networks", Computer Science \& Information Technology, CS \& IT-CSCP 2012

[8] S. Haykin, "Cognitive radio: Brain-empowered wireless communications," IEEE J. Sel. Areas Commun., vol. 23, no. 2, pp. 201-220, Feb.2005.

[9] J.M. García-Campos, D. G. Reina, S. L. Toral, F. Barrero, N. Bessis, E. Asimakopoulou and R. Hill, "Performance Evaluation of Reactive Routing Protocols for VANETs in Urban Scenarios Following Good Simulation Practices", 9th International Conference on Innovative Mobile and Internet Services in Ubiquitous Computing, 2015.

[10] Padmalaya Nayak and Pallavishree Sinha, "Analysis of Random Way Point and Random Walk Mobility Model for Reactive Routing Protocols for MANET Using NetSim Simulator", 3rd International Conference on Artificial Intelligence, Modelling and Simulation, 2015.

[11] V. Mandhare V. and R. C. Thool, "Comparing the Performance of Proactive and Reactive Routing Protocol in Mobile Ad-Hoc Network", International Conference on Industrial Instrumentation and Control (ICIC) College of Engineering Pune, India. May 28-30, 2015.

[12] L. Mejaele and E. O. Ochola, "Effect of Varying Node Mobility in the Analysis of Black Hole Attack on MANET Reactive Routing Protocols", IEEE, 2016.

[13] DaSilva, L. A. and Guerreiro, I., "Sequence-Based Rendezvous for Dynamic Spectrum Access," in Proc. of IEEE DySPAN, pp. 1-7, October 2008.

[14] Kondareddy, Y. R. and Agrawal, P., "Synchronized MAC Protocol for multi-hop cognitive radio networks," in Proc. of IEEE Intl. Conf. on Comm. (ICC), pp. 3198-3202, May 2008.

[15] Zhao, J., Zheng, H., and Yang, G.-H., "Spectrum Agile Radios: Utilization and Sensing Architecture," in Proc. of IEEE International Symposium on Dynamic Spectrum Access Networks, pp. 259-268, November 2005.

[16] M. Gerla, 'Fisheye state routing protocol (FSR) for ad hoc networks, Internet Draft, draft-ietf-manet-aodv03.txt, work in progress, 2002.

[17] lajos hanzo and rahim tafazolli, "Asurvey of QoS Routing Solutions for Mobile Ad hoc Networks," IEEE Communications Surveys \& Tutorials, 2nd quarte, 2007. 
[18] James Bernsen and D. Manivannan,"Greedy Routing Protocols for Vehicular Ad Hoc Networks," Wireless Communications and Mobile Computing Conference IWCMC 08, vol. 632, no. 637, pp. 6-8, Aug. 2008.

[19] Jamal Toutouh, Enrique Alba, "Optimizing OLSR in VANETS with Differential Evolution: A Comprehensive Study," ACM DIVANet'11, Miami, Florida, USA., 2011.

[20] Sonam Jain and Sandeep Sahu, "Topology vs. Position based Routing Protocols in Mobile Ad hoc Networks: A Survey," International Journal of Engineering Research \& Technology (IJERT), vol. 1, Issue 3, May 2012.

\section{Authors' Profiles}

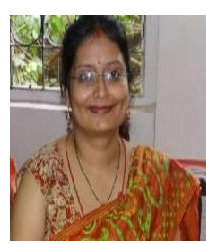

Shruti Singhroy (born September 23 1986) is a research scholar. She has Done her engineering in the field of electronics engineering and M.Tech in the field of VLSI. She is pursuing her $\mathrm{PhD}$ in Cognitive radio Wireless communication.

Dr. P. L. Zade presently working as a principal in Wardha Nagpur. He has done his Phd in the field of Antenna and microwave.

How to cite this paper: Shruti Singh Roy, P. L. Zade," Performance Optimization of Ad Hoc on Demand Vector Routing Protocol in Cognitive Radio Network", International Journal of Wireless and Microwave Technologies(IJWMT), Vol.8, No.3, pp. 23-32, 2018.DOI: 10.5815/ijwmt.2018.03.03 